\title{
Will 'green' aspects of dried blood spot sampling accelerate its implementation and acceptance in the pharmaceutical industry?
}

\author{
"The broader application of DBS in pharmacokinetic analysis is an excellent example of a 'green' technique based on \\ the benefits in reduction of animals, chemicals, labware and energy consumption."
}

Keywords: dried blood spot $\equiv$ drug development $\approx$ green chemistry $\approx$ microsampling $\approx$ pharmacokinetics $\approx$ toxicokinetics

Green chemistry is a philosophy of chemical research and engineering that encourages the design of products and processes minimizing the use and generation of hazardous substances [101]. Dried blood spot (DBS) technology has existed for over 40 years [1], but has recently gained attention as a microsampling technology for nonclinical and clinical pharmacokinetic analysis $[2,3]$. Method development and validation, and subsequent sample analysis, have been successfully carried out using LC-MS/MS and other detection methods for both large and small molecules. The concept of DBS has been extended from blood to plasma [4] and cerebrospinal fluid [5]. The broader application of DBS in pharmacokinetic analysis is an excellent example of a 'green' technique based on the benefits in reduction of animals, chemicals, labware and energy consumption. Many of these benefits have fostered the interest in, and in some cases the use of, this technology in the pharmaceutical industry. However, progress has been slow and the question remains: will the green aspects of DBS sampling accelerate its implementation and acceptance?

The advantages of DBS for preclinical studies include the well-recognized ethical benefits and associated resource savings. In DBS sampling, blood samples of 10-20 $\mu$ l are spotted on cellulose paper. Typically, four DBS spots are collected at each time point, requiring a total of approximately 50-100 $\mu \mathrm{l}$ of blood compared with approximately $500 \mu \mathrm{l}$ of blood typically collected for traditional plasma. The approximately five- to ten-fold reduction in blood volume results in:

- Reduction of animal usage. Depending on the study design, a $50 \%$ reduction in the number of rodents can be realized either by reduced numbers/group or elimination entirely of toxicokinetic satellite animals in toxicological study when samples are collected in DBS matrix [6];

- Reduction of sampling stress (from reduced volume in rodents as well as modified techniques of sample collection in non-rodents) [6];

- Improved data quality in rodents due to better correlation of exposure to test-article-related effects with serial sampling.

Therefore, DBS technology clearly addresses the reduction and refinement aspects of the $3 \mathrm{R}$ principle (i.e., reduction, refinement and replacement). Associated material resource savings include reduction in rodent housing and husbandry resources, which can be important for longer term chronic studies in particular, and reduction in test-article amount required in rodent studies, the benefits of which span the length of the development process. Less testarticle requirements during early stages of drug development, where test article availability is limited, can be particularly critical.

Additional material resource savings can result from the reduced sample volume and the streamlined sample processing. Sample collection for traditional plasma analysis requires an initial glass or plastic whole-blood collection tube, centrifugation and another plasma collection tube. Sampling for DBS eliminates the original whole-blood tube, replacing it with a small $(\sim 10-100 \mu \mathrm{l})$ capillary tube, for temporary transfer to the DBS paper, no centrifugation and the plasma tube is replaced with a

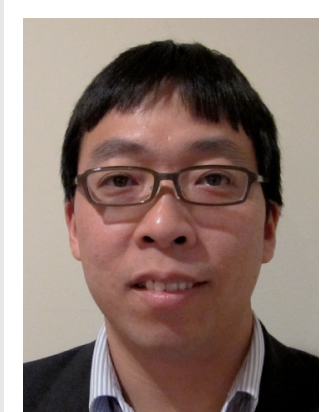

Fumin Li

Author for correspondence: Covance Laboratories, Inc., Bioanalytical Chemistry, 3301 Kinsman Boulevard, Madison, WI 53704, USA

E-mail: fumin.li@covance.com

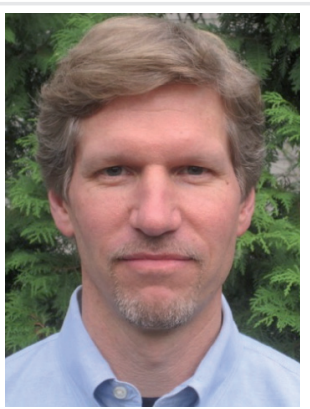

Stephen Ploch

Covance Laboratories, Inc., Nonclinical Safety Assessment, 330I Kinsman Boulevard, Madison, WI 53704, USA 
renewable resource (the cellulose DBS card). Sample processing for DBS can be more time consuming, particularly without automation, and requires a paper-punching device not needed for liquid-sample handling. Once the sample is obtained from the DBS card, the resources needed for bioanalytical sample processing and analysis are equivalent to traditional methods.

The DBS toxicokinetic technique can be continued through clinical studies, where the primary benefits include: enhancement of patient comfort via finger/heel pricking, instead of venipuncture; applicability to pediatric/juvenile studies, where blood volume is limited; and, use in remote geographical areas where refrigeration is difficult. Use of DBS has been estimated to provide real financial benefits in practice [7]. Much of the cost savings are a result of the stability of samples on the cards under room temperature conditions (sample storage/shipping). Compared with conventional liquid matrices such as plasma, DBS sampling does not require wet ice for temporary blood storage, centrifugation to harvest plasma, freezers $(-20$ or $-70^{\circ} \mathrm{C}$ ) to store processed samples and dry ice for sample shipment. The following are examples of estimated cost savings offered by DBS:

- According to Spooner, DBS microsampling has resulted in cost savings estimated by GlaxoSmithKline in the region of US $\$ 12$ million [8];

- Evans et al. found that the ability to ship and store DBS samples under ambient conditions can lead to cost savings of greater than $\$ 1$ million per year for a large organization, such as Sanofi, based on some broadly applicable assumptions [9];

- Cost savings in global clinical trials: the costs savings of DBS shipments compared with plasma can be $\$ 200$ per shipment and for a complicated trial the savings can add up to multiples of $\$ 100,000$ [10];

- Cost savings in sample storage: for a typical plasma sample, its life span can vary depending upon specific protocol requirements and study length. In general, a plasma sample stays in a freezer for at least 1 year before the study is finalized. Our in-house studies estimate that it costs roughly $\$ 1$ per year to store one plasma sample in a $-70^{\circ} \mathrm{C}$ freezer.

Some may argue that the cost savings from DBS sample shipping and storage is insignificant compared with the expense of a clinical trial, which can cost approximately $\$ 100$ million. Nevertheless, DBS offers something beyond simple economics. First, room temperature sample storage and shipping are of enormous value in preserving sample integrity. There is a risk associated when samples are shipped and stored at a low temperature $\left(-20\right.$ or $\left.-70^{\circ} \mathrm{C}\right)$, especially for compounds with temperature-sensitive stability. The temperature a sample goes through from collection to storage to analysis is normally not recorded. Any incident of subjecting samples to a temperature outside the range of stability during the long chain of sample custody can ruin sample integrity. The cost associated with the loss of reportable data or doubt about the integrity of data from a clinical study can be large in both money and time. In comparison, DBS samples largely eliminate the risk of losing sample integrity owing to temperature fluctuations (provided the stability has been properly established). Furthermore, DBS provides a unique capability to enhance stability of certain compounds [11]. When the matrix has been spotted on certain cellulose papers, bacteria are killed and enzymes are deactivated. As a result, analytes with instability caused by enzymatic degradation can exhibit enhanced stability in DBS. This can eliminate the need for the use of sometimes hazardous additives to stabilize metabolites in liquid matrices. Moreover, DBS can be an enabling technology for multisite clinical trials for certain diseases involving underdeveloped and/ or remote areas, where low temperature sample storage and shipping are not viable.

\section{"We are optimistic that the technical hurdles associated with DBS can be resolved with continued research."}

ㄸำ

The question therefore remains: will the green aspects of DBS sampling accelerate its implementation and acceptance in pharmaceutical industry? The answer depends on a number of factors. Technical challenges and regulatory uncertainty have slowed its recent progress. Compound-specific technical challenges include: determination of the impact of hematocrit; storage conditions; paper substrate and analyte extractability on the accurate assessment of drug exposure; sensitivity challenges encountered in LC-MS/MS assay development; and slow acceptance of kinetic data generated from blood as opposed to plasma. We are optimistic 
that the technical hurdles associated with DBS can be resolved with continued research. Although some of these technical challenges are widely understood, compound-specific, broad regulatory acceptance has been slow and led to some level of uncertainty. Unlike liquid matrices, there is no existing regulatory guideline for DBS method validation and sample analysis. Regulatory agencies understandably have taken a cautious approach (e.g., reportedly requiring parallel analysis of liquid whole blood in addition to DBS in certain circumstances). As with any new technology, this may be interpreted not as a setback for DBS microsampling but instead is a logical step in the process by which regulatory agencies can learn from real data and work toward forming appropriate and broad-based regulatory guidelines.

Whether DBS will become a niche technology or a more broadly usable one in the pharmaceutical industry is yet to be determined. Nevertheless, it is clear that the application of DBS as a means of sample collection shows promise on multiple fronts. It is likely that the great power of DBS is yet to be unlocked. To give DBS technology a fair opportunity, pharmaceutical companies should be encouraged to use DBS when appropriate for study design and compound characteristics. The scientific community should continue to improve the technology, conducting the best science and harmonizing the best practices. It is imperative that an open forum is maintained between industry stakeholders and regulatory agencies to gain mutual understandings of the technology. The ultimate goal is to use DBS sampling to produce valid data to support regulatory filings. The green aspects of DBS technology can and have provided a certain level of encouragement to accelerate its implementation and acceptance in the pharmaceutical industry, but the technology will only become more widespread if the data generated are accepted and valid. We think DBS is not just another cliché green technology.

\section{Acknowledgements}

The authors would like to thank F Kirchner and D Fast for their helpful discussions and critical review of the manuscript.

\section{Financial \& competing interests disclosure}

The authors have no relevant affliations or financial involvement with any organization or entity with a financial interest in or financial conflict with the subject matter or materials discussed in the manuscript. This includes employment, consultancies, honoraria, stock ownership or options, expert testimony, grants or patents received or pending, or royalties.

No writing assistance was utilized in the production of this manuscript.

\section{References}

1 Guthrie R, Susi A, A Simple phenylalanine method for detecting phenylketonuria in large populations of newborn infants. Pediatrics 32, 338-343 (1963).

2 Spooner N, Lad R, Barfield M. Dried blood spots as a sample collection technique for the determination of pharmacokinetics in clinical studies: considerations for the validation of a quantitative bioanalytical method. Anal. Chem. 81, 1557-1563 (2009).

3 Dainty TC, Richmond ES, Davies I, Blackwell MP. Dried blood spot bioanalysis: an evaluation of techniques and opportunities for reduction and refinement in mouse and juvenile rat toxicokinetic studies. Int. J. Toxicol. 31, 4-13 (2012).

4 Barfield M, Wheller R. Use of dried plasma spots in the determination of pharmacokinetics in clinical studies: validation of a quantitative bioanalytical method. Anal. Chem. 83, 118-124 (2011).

5 Rago B, Liu J, Tan B, Holliman C. Application of the dried spot sampling technique for rat cerebrospinal fluid sample collection and analysis. J. Pharm. Biomed. Anal. 55, 1201-1207 (2011).

Burnett JE. Dried blood spot sampling: practical considerations and recommendation for use with preclinical studies. Bioanalysis 3, 1099-107 (2011).

7 Beaudette P, Bateman KP. Discovery stage pharmacokinetics using dried blood spots. J. Chromatogr. B 809, 153-158 (2004).

8 Howard H. The 19th International Reid Bioanalytical Forum. Bioanalysis 3, 2709-2715 (2011).
9 Evans CA, Bruce C, Emmons GT et al. Conference report: DIA/PhRMA workshop on DBS sampling in the pharmaceutical industry: methodology, implementation \& best practices. Bioanalysis 2, 1355-1359 (2010).

10 Amsterdam P, Waldrop C. The application of dried blood spot sampling in global clinical trials. Bioanalysis 2, 1783-1786 (2010).

11 Bowen CL, Hemberger MD, Kehler JR, Evans CA. Utility of dried blood spot sampling and storage for increased stability of photosensitive compounds. Bioanalysis 2, 1823-1828 (2010).

\section{- Website}

101 US Environmental Protection Agency. www.epa.gov/greenchemistry 